\title{
Evaluation of Acute Toxicity and Dosimetric Parameters in High Risk Prostate Cancer Patients Treated by High Radiation Doses
}

\author{
Mohamed A. Daoud1,2, Yasser M. Saleh1,3*, Mohamed Elsherbini3 ${ }^{3,4}$, Mostafa Al Etreby ${ }^{2,5}$ \\ ${ }^{1}$ Department of Clinical Oncology and Nuclear Medicine, Mansoura Faculty of Medicine, Mansoura University, Mansoura, Egypt \\ ${ }^{2}$ Department of Oncology, Fakeeh Hospital, Jeddah, Saudi Arabia \\ ${ }^{3}$ Department of Oncology, Saudi-German Hospital Group, Abha, Saudi Arabia \\ ${ }^{4}$ Department of Clinical Oncology and Nuclear Medicine, Faculty of Medicine, Menofia University, Menofia, Egypt \\ ${ }^{5}$ Department of Clinical Oncology and Nuclear Medicine, Kasr Alainy Faculty of Medicine, Cairo, Egypt \\ Email: *dr_yasserms@yahoo.com
}

How to cite this paper: Daoud, M.A., Saleh, Y.M., Elsherbini, M. and Al Etreby, M. (2019) Evaluation of Acute Toxicity and Dosimetric Parameters in High Risk Prostate Cancer Patients Treated by High Radiation Doses. Journal of Cancer Therapy, 10, 654-670.

https://doi.org/10.4236/jct.2019.108054

Received: June 21, 2019

Accepted: August 18, 2019

Published: August 21, 2019

Copyright ( 2019 by author(s) and Scientific Research Publishing Inc. This work is licensed under the Creative Commons Attribution International License (CC BY 4.0).

http://creativecommons.org/licenses/by/4.0/

\section{(c) (i) Open Access}

\begin{abstract}
For high risk prostate cancer, the treatment volumes and even dose levels are still a controversial issue. The aim of this study is to evaluate the dosemetric parameters and acute toxicity of dose-escalated whole pelvis (WP) Intensity Modulated Radiation Therapy (IMRT) and volumetric modulated arc therapy (VMAT) prostate boost following neoadjuvant and concomitant with androgen deprivation therapy in high-risk prostate cancer patients. This analysis included 73 high-risk prostate cancer patients treated with WP-IMRT followed by boost to the prostate by VMAT to total dose of 80 Gy; between January 2014 and October 2016. Androgen deprivation therapy (ADT) was given for all patients before and during radiation therapy. Drawing the dose volume histograms (DVHs) was done for planning target volumes (PTVs), including Prostate PTV \& nodal PTV, and organs at risk including rectum, bladder, femoral heads, and bowel bag for the plans. Acute radiation toxicities were reported during the radiation course and the following 3 months. The DVH analysis showed good coverage of PTVs and organs at risk doses were acceptable. No recorded acute Grade $\geq 3$ toxicity. Acute grade 1 toxicity for Gastrointestinal (GI) and Genitourinary (GU) were $65 \%$ and 35\% respectively, while Grade 2 toxicity was 30\% for both. The Proctitis and frequency were the commonest acute toxicity and were maximal during the 5th week of radiation therapy. Dose escalation in two phases utilizing Simultaneous integrated boost (SIB) combined with ADT in high risk prostate cancer patient is feasible and associated with acceptable acute GI and GU toxicity.
\end{abstract}




\section{Keywords}

Radiation Therapy, Dose Escalation, Cancer Prostate, Androgen Deprivation Therapy

\section{Introduction}

Dose-escalated radiation therapies (80 Gy and higher) in high-risk prostate cancer patients have demonstrated improvement in outcome and biochemical disease-free survival [1] [2] [3]. In a Phase III trial, The Radiation Therapy Oncology Group (RTOG) showed improved progression-free survival (PFS) for high-risk prostate cancer patients treated with Whole Pelvis Radiation Therapy (WPRT) compared with prostate-only radiation therapy (PORT) [4]. Also, an updated analysis of the same study demonstrated improvement of PSA control and PFS at 10 years with added neoadjuvant hormonal therapy to WPRT [5]. Hence, there is increased interest in radiation dose escalation combined with androgen deprivation in high risk prostate cancer patients [6] [7]. An ongoing GETUG-AFU-18 phase III trial is evaluating the impact of dose escalation in combination with 3-year androgen deprivation treatment on 5-year biochemical or clinical control in high-risk prostate cancer patients [8]. Dose escalation can be achieved with either 3-Dimensional Conformal Radiation Therapy (3-DCRT) or with intensity-modulated Radiation Therapy (IMRT). Previous studies demonstrated the superiority of IMRT over the conventional radiation techniques for WPRT in sparing of organs at risk [9] and superior target coverage [10]. In an analysis using SEER data showed that patients treated with IMRT were less likely to have physician reported gastrointestinal morbidity compared to those treated with 3-DCRT but more likely to have erectile dysfunction [11]. Furthermore, recent clinical trials confirmed that WP-IMRT had acceptable rates of acute toxicity [12] [13] [14]. IMRT permits the use of different total doses and different doses/fraction to different volumes within the irradiation field, utilizing the "simultaneous integrated boost" (SIB) technique. Consequently, the IMRT-SIB technique allows different doses to the prostatic area and the pelvic lymph nodes. However, in the context of dose escalation to the prostate, dosimetric and clinical results from the literature comparing WP IMRT with PO IMRT are still limited. In a planning study, Guckenberger demonstrated similar toxicity risks for rectum, bladder and small bowel in both WP IMRT and PO IMRT [15]. Volumetric Modulated Arc Therapy (VMAT) Provides excellent dose distribution with less treatment time and monitor units. Planning studies on dosimetric comparison of the prostate only demonstrated that VMAT provided equal or better target coverage and normal tissue sparing over IMRT [16] [17] [18].

The aim of current study is to assess the dosimetric parameters and acute toxicity of dose-escalated WP-IMRT and VMAT prostate boost combined with neoadjuvant and concurrent androgen deprivation therapy in high risk patients of prostate cancer. 


\section{Materials and Methods}

\subsection{Patients}

The study included a cohort of 73 high-risk prostate cancer patients treated in two hospitals with WP-IMRT followed by prostate boost by VMAT to total dose of 80 Gy between January 2014 and October 2016. All patients had locally advanced disease with no distant metastasis and not suffering from other malignant disease. In this analysis, we aimed at evaluation of the dosimetric parameters for the dose escalation and its impact on the acute toxicity when combined with androgen deprivation therapy. Recording the grades of acute toxicity for this combined treatment modality was our primary outcome. All patients were diagnosed by trans-rectal ultrasound-guided core biopsy, 12 cores were obtained for each patient. High-risk was defined as CT3/4 N0 M0, according to the 2010 American Joint Committee on Cancer staging classification [19], and/or a Gleason score of $\geq 8$ and/or a pretreatment PSA concentration of $\geq 20 \mathrm{ng} / \mathrm{ml}$. The local institutional ethics committee of Fakeeh Hospital approved the study. Patient characteristics are shown in Table 1. All patients received androgen deprivation therapy (ADT), starting 4 - 6 months before Radiation Therapy (RT) and continued for a total period of $\geq 24$ months.

\subsection{Simulation Organ Contouring and Planning}

Computed tomography (CT) was acquired in the supine position, with 2-mm slices thickness from the dome of diaphragm to about $5 \mathrm{~cm}$ below the ischial tuberosities. Immobilization was obtained using Headrest, kneefix and feetfix (CIVCO Medical Solutions, Coralville, IA). Before CT simulation patients were instructed to have a comfortably filled bladder, by drinking one litter of water, and an empty rectum. The CT data set was transferred to the Eclipse ver. 13.6 treatment planning system (Varian Medical Systems, Palo Alto, CA). The prostate clinical target volume (CTV) was defined as the entire prostate and the seminal vesicles, and any visible tumor extension. The prostate planning target volume (PTV) was generated by adding $10-\mathrm{mm}$ margin to the prostate CTV in all dimensions, except posteriorly, where a $6-\mathrm{mm}$ margin was used. Based on the consensus recommendations of the RTOG [20], the nodal CTV consisted of a 0.7-cm expansion volume on the obturator vessels, the common iliac, external and internal iliac vessels, while excluding adjacent bone, muscle, bowel and bladder. The nodal CTV commenced at the level of L5 to S1 interspace, with volumes of the external iliac nodal stopping at the top of the femoral head and the obturator nodal volumes stopping just above the symphysis pubis. The presacral nodes were included in the nodal CTV down to S3-S2 interspace. The nodal PTV was defined by adding $0.3-\mathrm{mm}$ expansion of the nodal CTV. For the prostate boost, the CTV included the prostate and proximal $6-8 \mathrm{~mm}$ of the seminal vesicles. The PTV boost was generated by adding $6 \mathrm{~mm}$ margin to the CTV boost except $5 \mathrm{~mm}$ posteriorly. Contouring of the (Organs at Risk) OAR followed the RTOG pelvic normal tissue contouring guidelines [21]. The rectum 
Table 1. Patients characteristics.

\begin{tabular}{|c|c|c|}
\hline Character & Value & Percent \\
\hline \multicolumn{3}{|l|}{ Age } \\
\hline Median & 65 & \\
\hline Range & $57-86$ & \\
\hline \multicolumn{3}{|l|}{ Performance status } \\
\hline 0 & 43 & 58.9 \\
\hline 1 & 30 & 41.1 \\
\hline \multicolumn{3}{|l|}{ Gleason score } \\
\hline$\leq 6$ & 7 & 9.6 \\
\hline 7 & 23 & 31.5 \\
\hline$\geq 8$ & 43 & 58.9 \\
\hline \multicolumn{3}{|l|}{ Biopsy core $\%$} \\
\hline$<50 \%$ & 15 & 20.6 \\
\hline$\geq 50 \%$ & 58 & 79.4 \\
\hline \multicolumn{3}{|l|}{ PSA (ng/ml) } \\
\hline Median & 28 & \\
\hline Rang & $13-300$ & \\
\hline \multicolumn{3}{|l|}{ Clinical Stage } \\
\hline $\mathrm{T} 1$ & 14 & 19.2 \\
\hline $\mathrm{T} 2$ & 7 & 9.6 \\
\hline T3 & 44 & 60.3 \\
\hline $\mathrm{T} 4$ & 8 & 11 \\
\hline Diabetes & 29 & 39.7 \\
\hline Anticoagulant therapy & 14 & 19.2 \\
\hline \multicolumn{3}{|c|}{ Androgen deprivation therapy (ADT) } \\
\hline$\leq 24$ months & 59 & 80.8 \\
\hline$>24$ months & 14 & 19.2 \\
\hline
\end{tabular}

was contoured from the level of the ischial tuberosities to the recto-sigmoid flexure, and the whole bladder was contoured from its apex to the dome. Both femoral heads were delineated to the level of the ischial tuberosities. The bowel bag was contoured as the entire volume of peritoneal space down to level of S1. The treatment plan was given in two phases. In the first phase, the nodal PTV and the prostate PTV received 48.6 Gy and 54 Gy, respectively, both in 27 fractions. IMRT with a simultaneous integrated boost (SIB) technique was selected in phase one treatment. Nine co-planner fields are aligned equal-spaced in $360^{\circ}$ around the patient $(0,40,80,120,160,200,240,280$, and 320). Planning risk volumes PRVs were created for rectum and bladder to exclude from the high dose region. Other helping contours (Ring structures with $0.3 \mathrm{~cm}$ internal margin and $3 \mathrm{~cm}$ external margin) were created around the nodal and prostate PTVs separately for better control the dose fall off beyond each PTV. A set of dose constrains were defined for the PTVs and the OARs, and no normalization method was selected for any IMRT plan. Two lower limits were defined for each PTV as $100 \%$ and $97 \%$ of the volume and prescribed to $95 \%$ and $100 \%$ of the 
dose; also two upper limits, $2 \%$ and $0.1 \%$ of the volume were defined as $101 \%$ and $103 \%$ of the prescribed dose respectively. By using these constrains and through the interactive optimization process, the mean and median dose for each PTV is usually kept equal to the corresponding prescribed dose. Smoothing Objectives were also used to have smoother fluence in the $\mathrm{x}$-direction to ensure minimal MU factor. In the second phase, the prostate PTV received $26 \mathrm{~Gy}$ in 13 fractions using double-arc VMAT clock wise and counter clockwise (CW \& CCW). Control points for each arc were adjusted to give at least 1.5 angle step resulting in 178 control points. Variable collimator angle was defined for each arc to minimize the tongue and groove effect. Both phases were optimized using photon optimization algorithm (PO) newly developed in Eclipse V16.0.03. Treatment Plans were considered acceptable when $\geq 95 \%$ of the PTV received $\geq$ $95 \%$ of the prescribed dose. For the OAR dose volume constraints were: Rectal mean dose less than $50 \mathrm{~Gy}$, minimal dose of $70 \mathrm{~Gy}$ (V70Gy) less than $15 \%$ and V50Gy less than 45\%; and V70Gy less than 25\% and V50Gy less than 50\% for the bladder. For the femoral heads, the maximal point dose was less than $55 \mathrm{~Gy}$ and minimal dose to $2 \%$ (D2\%) less than 50 Gy. For the bowel bag, V45Gy was less than $195 \mathrm{ml}$. The dose calculation was performed using the anisotropic analytic algorithm (AAA, version 16.0.03) and a voxel size of $0.25 \times 0.25 \times 0.25 \mathrm{~cm}^{3}$.

\subsection{Image Guidance}

Online image-guided radiotherapy, patients were treated with a Traiology treatment unit (Varian Medical Systems, USA). Daily KV image guidance with On Board Imaging (OBI) and bi-weekly cone beam CT (CBCT) was performed in all patients. In the initial set up, the patients were immobilized in Headrest, kneefix and feetfix; the skin marks on the patient were used after applying shift. Orthogonal kilovoltage radiographs of the patients were then obtained using the OBI and registered to the reference digitally reconstructed radiographs (DRR) generated from the planning CT. Once the bone registration was well adjusted, CBCT images were also performed and used to obtain the target/soft tissue registration.

\subsection{Dosimetric Analysis}

Dose-volume histograms (DVHs) were constructed for the prostate PTV, nodal PTV, rectum, bladder, femoral heads, and bowel bag in each plan. Parameters chosen for measuring dosimetric quality of treatments were D95\% and D2\% for the prostate PTV and for nodal PTV were mean dose and D95\%. For the rectum and bladder, the analysis included the (Maximum Dose) D max, mean dose, V75Gy, V70Gy, V65Gy and V50Gy. For the bowel bag and femoral heads V45Gy and D max were measured respectively.

\subsection{Acute Radiation Toxicity}

All patients were checked weekly during radiation therapy and two weeks following radiation then monthly thereafter. Acute toxicity is reported during radiation and in the first 3 months following treatment using the Common Termi- 
nology Criteria for Adverse Events (CTCAE) version 4.0 adverse event scoring system. Dosimetric data for organs at risk in patients experienced Grade 1 or less toxicity were compared with those experienced Grade 2 toxicity.

\subsection{Statistical Analysis}

An unpaired Student's t test was used to compare mean values of each dosimetric parameter. Chi-square analysis was used for toxicity profile analysis. All statistical analyses were performed using the software SPSS version 22.0 (SPSS Inc., Chicago, IL, USA). All reported $\mathrm{P}$-values are two-tailed and $\mathrm{P}<0.05$ was considered statistically significant.

\section{Results}

All Patients received the prescribed dose with no interruption of treatment due to acute radiation toxicity. Androgen deprivation therapy (ADT) was given for all patients before and during radiation therapy. Seven patients continued their ADT post radiation to complete 24 months. The dose distribution in both axial and coronal plans for one patient is shown in Figure 1. The dosimetric values for the PTVs and Organs at risk (OAR) are illustrated in Table 2. All the

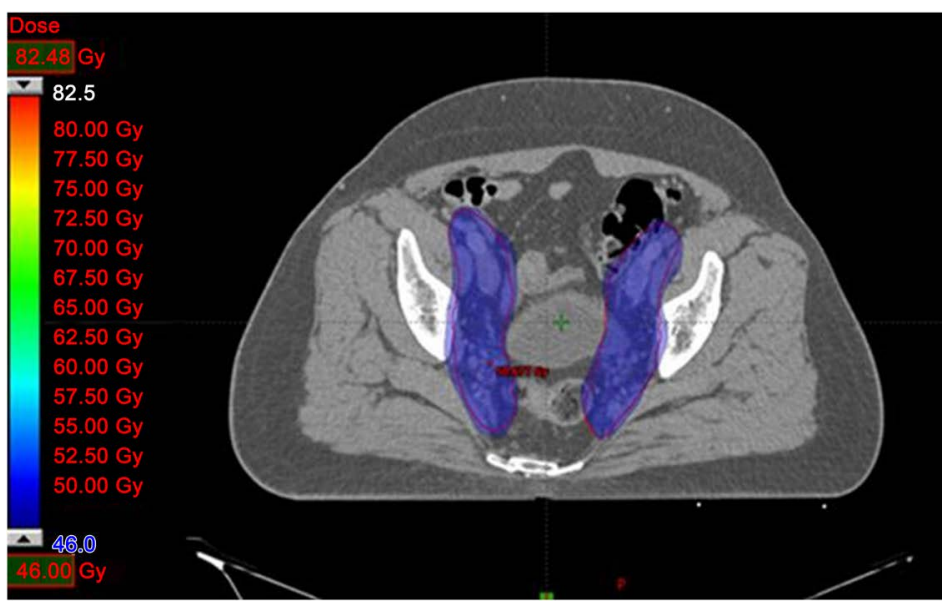

(a)

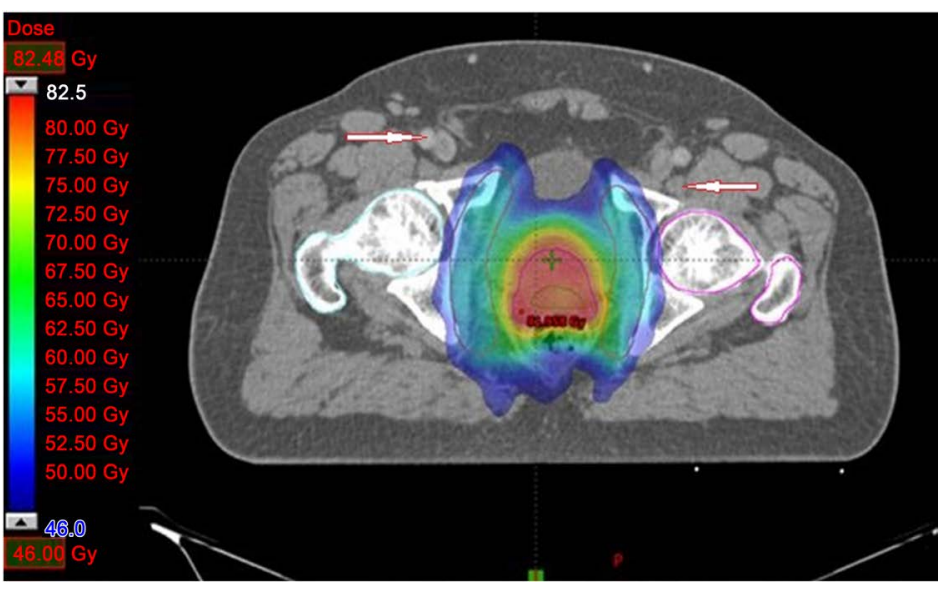

(b) 


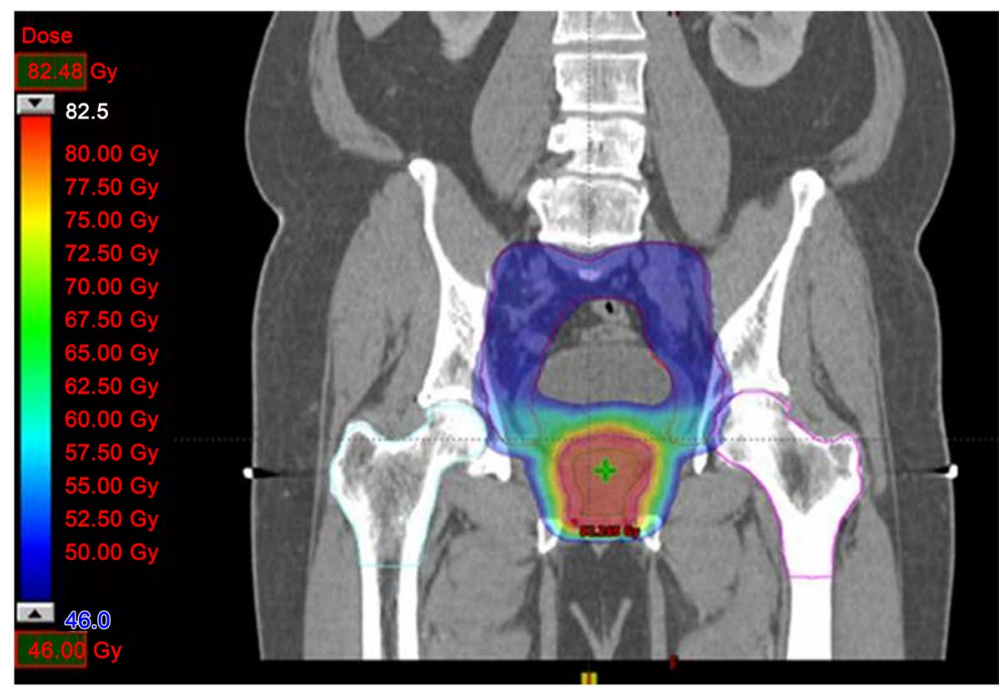

(c)

Figure 1. Dose distributions for composite plan. Axial computed tomography (CT) images of the (a) the pelvic lymph nodes (b) pelvis at the level of the prostate gland. Coronal CT showing the prostate gland and the pelvic lymph nodes (c). Dose color wash is from 46 Gy (dark blue) to $\sim 81 \mathrm{~Gy}$ (red).

Table 2. Dosimetric parameters.

\begin{tabular}{|c|c|c|c|}
\hline & Parameter & Unit & Mean \pm SD \\
\hline \multirow[t]{4}{*}{ Prostate PTV } & Volume & $\left(\mathrm{cm}^{3}\right)$ & $101.4(23.8)$ \\
\hline & $D_{\text {mean }}$ & (Gy) & 80 \\
\hline & $\mathrm{D}_{2 \%}$ & (Gy) & $81.5 \pm 0.8$ \\
\hline & $\mathrm{D}_{95 \%}$ & (Gy) & $79.4 \pm 0.7$ \\
\hline \multirow[t]{3}{*}{ Nodal PTV } & Volume & $\left(\mathrm{cm}^{3}\right)$ & $672.5 \pm 118.4$ \\
\hline & $D_{\text {mean }}$ & (Gy) & $52.1 \pm 2.1$ \\
\hline & $\mathrm{D}_{95 \%}$ & (Gy) & $48.6 \pm 0.9$ \\
\hline \multirow[t]{7}{*}{ Rectum } & Volume & $\left(\mathrm{cm}^{3}\right)$ & $112.3 \pm 72.9$ \\
\hline & $\mathrm{D}_{2 \%}$ & (Gy) & $78.8 \pm 1.3$ \\
\hline & $\mathrm{V}_{70 \mathrm{~Gy}}$ & $(\%)$ & $10.3 \pm 5.6$ \\
\hline & $\mathrm{V}_{65 \mathrm{~Gy}}$ & $(\%)$ & $14.3 \pm 6.9$ \\
\hline & $\mathrm{V}_{50 \mathrm{~Gy}}$ & $(\%)$ & $32.7 \pm 12$ \\
\hline & $\mathrm{V}_{45 \mathrm{~Gy}}$ & $(\%)$ & $47.2 \pm 12.2$ \\
\hline & $\mathrm{V}_{20 \mathrm{~Gy}}$ & $(\%)$ & $97.9 \pm 5.3$ \\
\hline \multirow[t]{6}{*}{ Bladder } & $\mathrm{D}_{\text {mean }}$ & (Gy) & $50.2 \pm 7$ \\
\hline & $\mathrm{D}_{2 \%}$ & (Gy) & $80.4 \pm 1.2$ \\
\hline & $\mathrm{V}_{80 \mathrm{~Gy}}$ & $(\%)$ & $4.6 \pm 4.9$ \\
\hline & $\mathrm{V}_{75 \mathrm{~Gy}}$ & $(\%)$ & $9.8 \pm 5.9$ \\
\hline & $\mathrm{V}_{70 \mathrm{~Gy}}$ & (\%) & $14.3 \pm 7.3$ \\
\hline & $\mathrm{V}_{65 \mathrm{~Gy}}$ & $(\%)$ & $19.5 \pm 9.7$ \\
\hline Left femoral head & $\mathrm{D}_{2 \%}$ & (Gy) & $42.8 \pm 3.3$ \\
\hline Right femoral head & $\mathrm{D}_{2 \%}$ & (Gy) & $43.4 \pm 2.6$ \\
\hline Bowel bag & $\mathrm{V}_{45 \mathrm{~Gy}}$ & $\left(\mathrm{~cm}^{3}\right)$ & $118.8 \pm 54.6$ \\
\hline
\end{tabular}


dosimetric parameters were satisfactory and acceptable. No grade 3 or 4 toxicities were reported and the genitourinary tract toxicities were more frequent in the patient during radiation therapy. In Figure 2, about $75 \%$ of patients developed
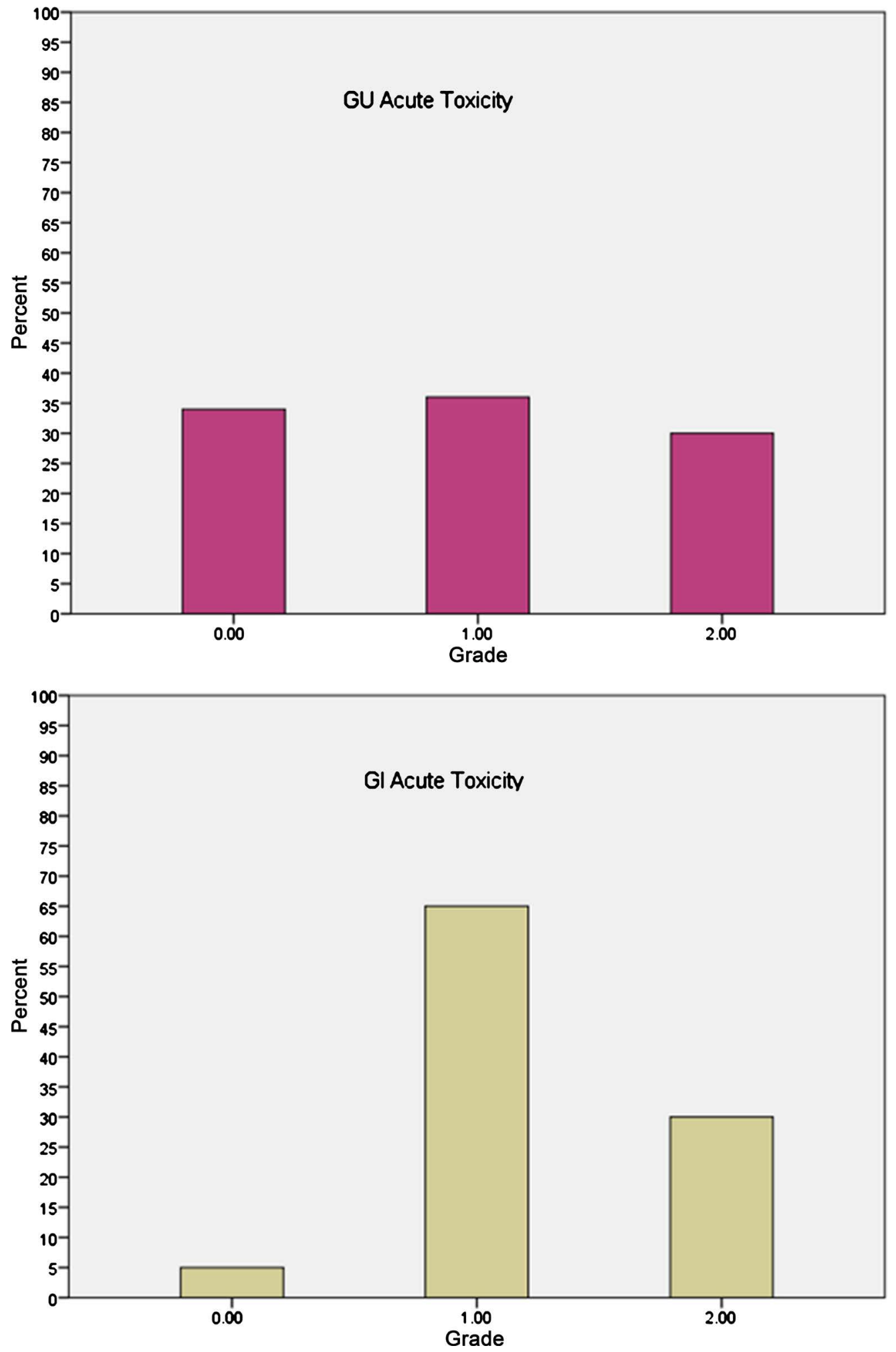

Figure 2. Incidence of acute gastrointestinal (GI) and genitourinary (GU) toxicity by grades. 
grade 2 acute GUT toxicities and 30\% developed Grade 2 GIT toxicities. Among the GUT toxicities frequency occurred in all treated patients with 51 (70\%) patients developed Grade 2 (Table 3). Also, all patients developed Proctitis and 15 (20.5\%) of them developed Grade 2 Proctitis. Acute diarrhea was reported in 65 patients and $48 \%$ of patient developed grade 1 diarrhea. The grade of toxicities increased gradually with progress of radiation therapy weeks. In Figure 3 and

Table 3. Acute toxicity profile and grades.

\begin{tabular}{|c|c|c|c|c|c|c|}
\hline \multirow{2}{*}{ Toxicity } & \multicolumn{2}{|c|}{ Grade 0} & \multicolumn{2}{|c|}{ Grade 1} & \multicolumn{2}{|c|}{ Grade 2} \\
\hline & No. & $\%$ & No. & $\%$ & No. & $\%$ \\
\hline GI & \multicolumn{2}{|c|}{$5 \%$} & \multicolumn{2}{|c|}{$65 \%$} & \multicolumn{2}{|c|}{$30 \%$} \\
\hline Proctitis & \multicolumn{2}{|c|}{0} & 58 & 79.5 & 15 & 20.5 \\
\hline Diarrhea & 8 & 11 & 35 & 48 & 30 & 41 \\
\hline GU & \multicolumn{2}{|c|}{$34 \%$} & \multicolumn{2}{|c|}{$36 \%$} & \multicolumn{2}{|c|}{$30 \%$} \\
\hline Frequency & \multicolumn{2}{|c|}{0} & 22 & 30.1 & 51 & 69.9 \\
\hline Incontinency & 37 & 50.7 & 28 & 38.4 & 8 & 11 \\
\hline Retention & 59 & 80.8 & 14 & 19.2 & \multicolumn{2}{|c|}{0} \\
\hline Urinary tract pain & 8 & 11 & 28 & 38.4 & 37 & 50.7 \\
\hline Urgency & 22 & 30.1 & 36 & 49.3 & 15 & 20.5 \\
\hline
\end{tabular}

GI: Gastrointestinal; GU: Genitourinary.

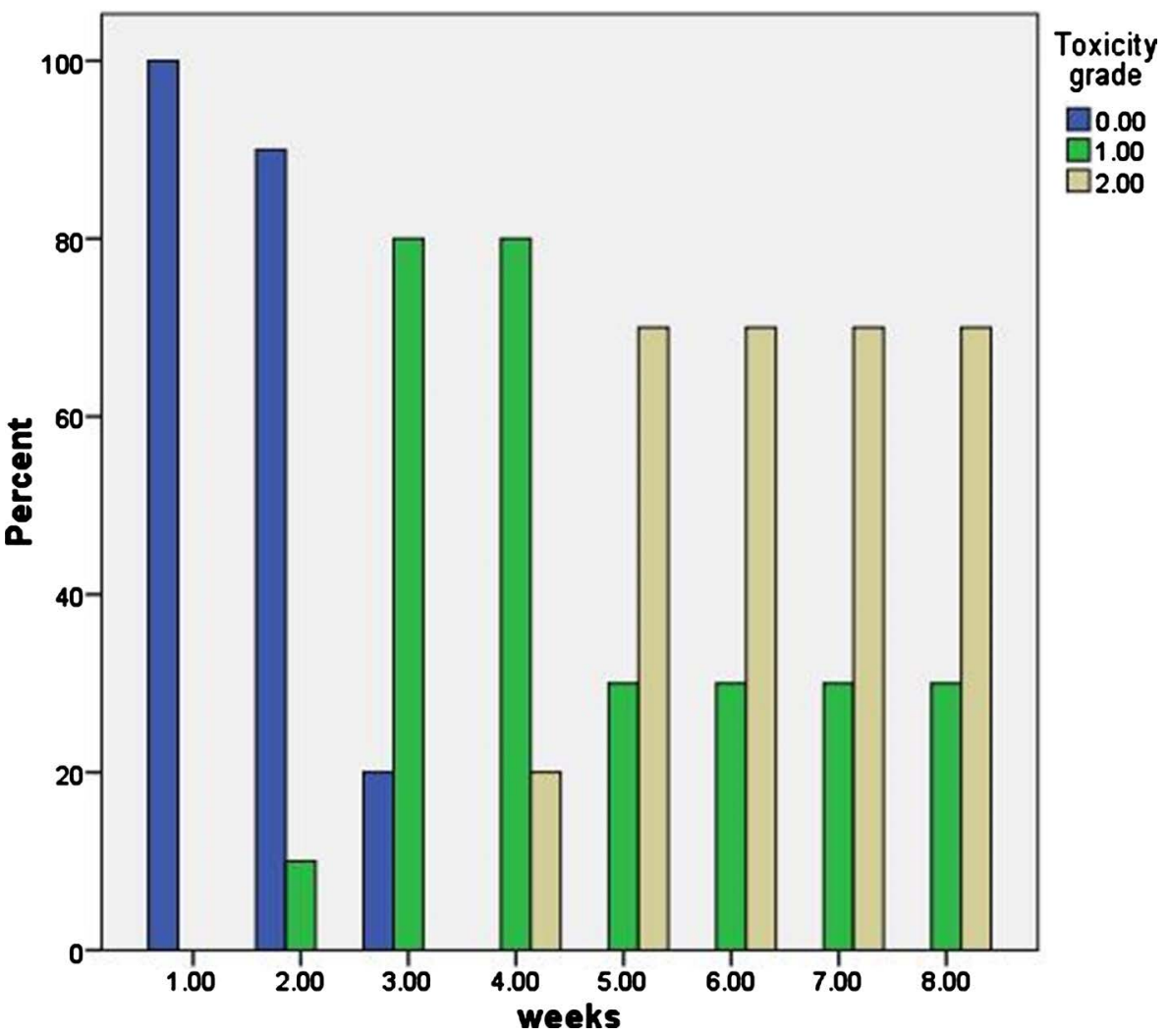

Figure 3. Prevalence of frequency grades during weeks of radiation therapy. 
Figure 4 the frequency increased in incidence and grade and became maximum (Grade 2) during the 5th week and continued till 8th week of radiation therapy. Acute diarrhea reached its maximum prevalence during the 5th week then decreased after that due to the end of whole pelvic irradiation. When the Grade of toxicity $\leq 1$, Grade 2 was compared in correlation with the different dose levels to the rectum, bladder and bowel bag (Table 4). There was no significant correlation with any dose level and the grade of toxicity.

\section{Discussion}

In patients with high-risk prostate cancer treatment volumes and even dose levels are still controversial issue [22]. Some investigators showed no difference between PORT and WPRT [23]. However, a major limitation of their study was the relatively small dose delivered of 72 Gy. A large phase III trial (RTOG 0924) is ongoing to answer the question of survival benefit of dose escalation WPRT combined with ADT in high risk patients [24]. Whole pelvic irradiation is often recommended in this setting, raising concerns about an increase in radiation toxicity to the organs at risk. The new technical developments such as SIB-IMRT, VMAT combined with IGRT have allowed radiation oncologists to achieve a better protection of risk organs while providing higher dose conformity to target volumes. In our study, all patients received ADT and dose escalation was done through 2 phases while maintaining the standard dose fractionation rang (1.8 - $2 \mathrm{~Gy})$ in order to obtain the highest possible cell killing effects. Dose

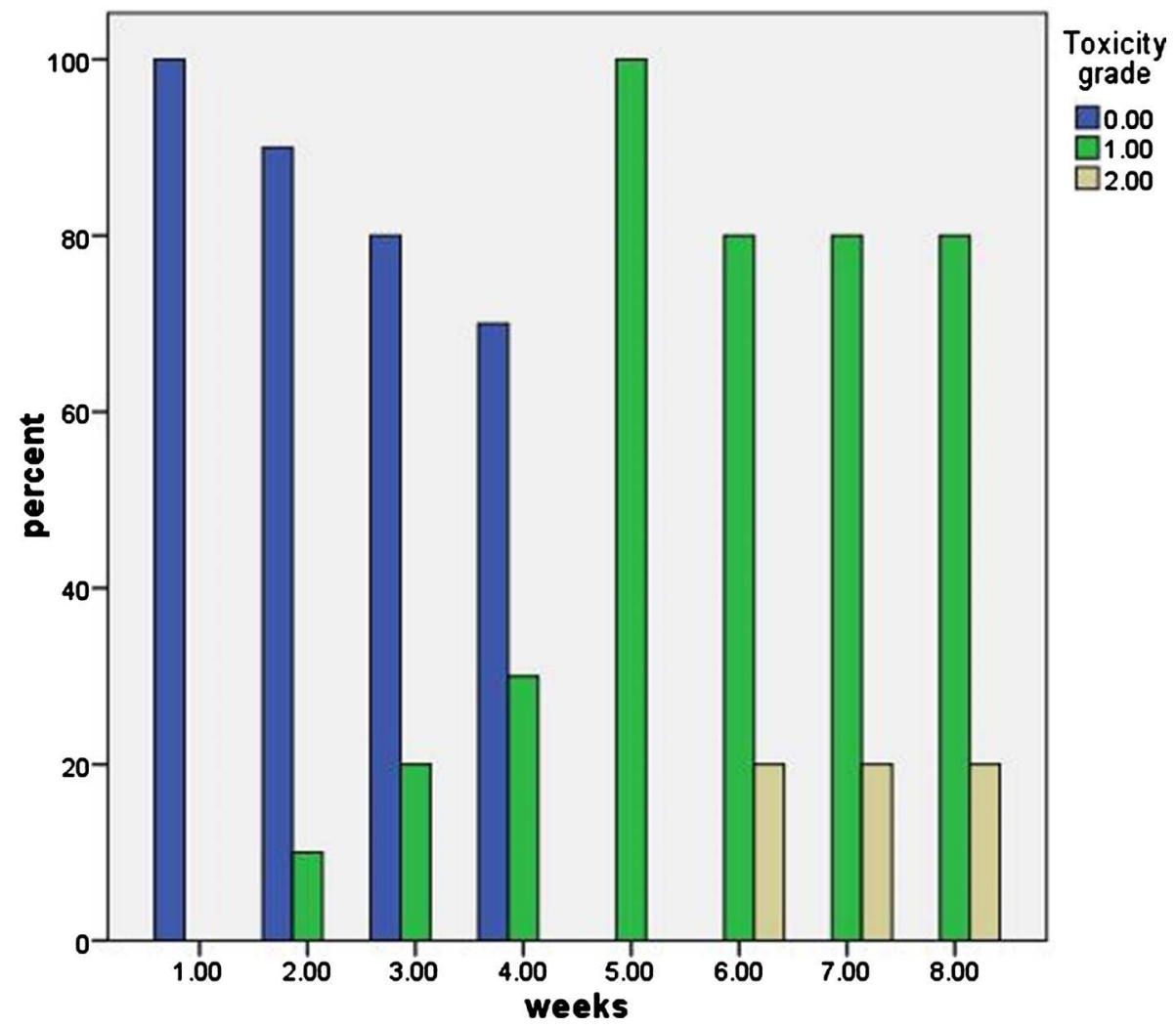

Figure 4. Prevalence of Proctitis grade during weeks of radiation therapy. 
Table 4. Comparison of dose values to the rectum, bladder, and bowel bag stratified by toxicity grades.

\begin{tabular}{|c|c|c|c|c|}
\hline Parameter & Unit & $\begin{array}{c}\text { Grade } \leq 1 \\
\text { Mean } \pm \text { SD }\end{array}$ & $\begin{array}{c}\text { Grade } 2 \\
\text { Mean } \pm \text { SD }\end{array}$ & $P$ Value \\
\hline \multicolumn{5}{|l|}{ Rectum } \\
\hline $\mathrm{D}_{2 \%}$ & (Gy) & $78.9 \pm 1.3$ & $78.7 \pm 1.1$ & 0.5 \\
\hline $\mathrm{V}_{75 \mathrm{~Gy}}$ & (\%) & $6.2 \pm 4.4$ & $5.4 \pm 4.6$ & 0.5 \\
\hline $\mathrm{V}_{70 \mathrm{~Gy}}$ & $(\%)$ & $10.5 \pm 5.3$ & $9.5 \pm 5.7$ & 0.7 \\
\hline $\mathrm{V}_{65 \mathrm{~Gy}}$ & (\%) & $14.3 \pm 6.5$ & $14.1 \pm 6.9$ & 0.8 \\
\hline $\mathrm{V}_{50 \mathrm{~Gy}}$ & (\%) & $32.9 \pm 11.2$ & $32.1 \pm 12.3$ & 0.8 \\
\hline $\mathrm{V}_{45 \mathrm{~Gy}}$ & (\%) & $47.4 \pm 12.2$ & $46.7 \pm 10.5$ & 0.8 \\
\hline $\mathrm{V}_{20 \mathrm{~Gy}}$ & (\%) & $97.5 \pm 5.5$ & $98.8 \pm 3.7$ & 0.3 \\
\hline \multicolumn{5}{|l|}{ Bladder } \\
\hline $\mathrm{D}_{\text {mean }}$ & (Gy) & $49.1 \pm 6.6$ & $51.1 \pm 6.9$ & 0.5 \\
\hline $\mathrm{D}_{2 \%}$ & (Gy) & $80.3 \pm 1.2$ & $80.6 \pm 0.9$ & 0.3 \\
\hline $\mathrm{V}_{80 \mathrm{~Gy}}$ & (\%) & $4.1 \pm 4.5$ & $4.5 \pm 5.2$ & 0.7 \\
\hline $\mathrm{V}_{75 \mathrm{~Gy}}$ & (\%) & $9.75 \pm 5.5$ & $10.0 \pm 6.1$ & 0.8 \\
\hline $\mathrm{V}_{70 \mathrm{~Gy}}$ & (\%) & $22.7 \pm 22.6$ & $18.3 \pm 15.8$ & 0.4 \\
\hline $\mathrm{V}_{65 \mathrm{~Gy}}$ & (\%) & $18.9 \pm 8.9$ & $20.8 \pm 10.1$ & 0.4 \\
\hline \multicolumn{5}{|l|}{ Bowel bag } \\
\hline $\mathrm{V}_{45 \mathrm{~Gy}}$ & $\left(\mathrm{~cm}^{3}\right)$ & $115.9 \pm 23.1$ & $125.9 \pm 34.7$ & 0.5 \\
\hline
\end{tabular}

escalation can be obtained in single phase by SIB to WPLNs with hypofractionation regimen to the prostate. However, the efficacy and late toxicity of this regimen still need investigations [25]. Furthermore, despite the total dose to the pelvis (50 - $52 \mathrm{~Gy}$ ) with $\alpha / \beta 1.5 \mathrm{~Gy}$, it is unlikely to eradicate subclinical or detectable LNs metastases that could have a different radiobiological behavior from the primary lesion, being more aggressive and showing a more cellular replication and metastatic potential. Based on this hypothesis, the $\alpha / \beta$ ratio to be taken in consideration for LN metastases is likely to be $1.8-2 \mathrm{~Gy}$. We chose the SIB-IMRT in phase I for the pelvic LNs and the prostate as this technique generated concave dose distributions and delivered radical doses to the pelvic nodes and prostate gland while reducing the dose to surrounding organs at risk. The same finding was reported before by Yoo et al. [26] in a dosimetric study comparing the treatment plans of ten patients with PTV including prostate, seminal vesicles, and lymph nodes. They showed that IMRT reached better dose sparing for bladder, rectum, and small bowel than did VMAT. On the other hand, Riou et al. [27] indicated a clinically and statistically significant reduction in doses delivered to the bladder, rectum, and small bowel when using VMAT in simultaneous integrated boost plans. However, this study used the single-phase SIB for dose calculation rather than two phases like our study and the Yoo study. Also, 
in the single-phase SIB, the dose per fraction for the PLNs was about 1.5 Gy and the aim of the study was to decrease the dose to OAR mainly. For the phase II (boost), we used the VMAT technique as it offered more dose homogeneity and conformity to the prostate and seminal vesicles with less treatment time and so decrease the chance of interfactional movements. The acute toxicity rates reported in the current study were compared favorably with those reported in other series that employed dose escalation WP-IMRT. Bayley et al., [14] reported grade II GI and GU toxicity of $31 \%$ and $44 \%$ respectively with total dose of 79.8 Gy/42 fractions. Also, Deville et al., [28] reported 50\% grade II toxicity for both GI and GT. On the other hand, Ishii et al., [29] reported fewer incidences of grade II toxicity for both GI and GU (16\% and 13\%) respectively; utilizing SIB-VMAT technique with total dose 78 Gy/39 fractions. None of our patients developed acute grade III toxicity indicating that dose escalation with this two-phase technique is very feasible in high risk prostate cancer. Some reports showed association between acute toxicity and development of subsequent late complications [30] [31]. Therefore, the acceptably low incidence of acute toxicity in the current study might contribute to decreasing the late side effects in further follow-up. In the current study, there was no correlation between the acute toxicity and the dosimetric parameters. The same finding was also observed by other investigators [29] [32] and [33]. This lack of correlation between toxicity and dosimetric variables could be due to the low dosimetric parameters and low frequencies of the severe acute toxicities. In this study, daily image guidance with OBI KV and biweekly CBCT was practiced in all patients. Ferjani et al. [34] demonstrated that pelvic SIB-IMRT for both PLNs and the prostate, with a planning margin to the prostate of $6-8 \mathrm{~mm}$ and a planning margin of $5 \mathrm{~mm}$ to the PLN, would result in good aligning to the prostate soft tissue on daily CBCT, but aligning to the pelvic bone would result in underdosing to the prostate in one-third of fractions. For that reason, we used $7 \mathrm{~mm}$ margin for PLNs, $10 \mathrm{~mm}$ as PTV margin for the prostate in phase one and $6 \mathrm{~mm}$ for boost. Also, all patients were adapted to have comfortably full bladder and rectum evacuated before each radiation treatment session to decrease the chance of prostate mobility. In the current study, IG was done without fiducial markers (FMs) and image registration was done based on both boney marks and soft tissues. Chung et al. [35] reported $13 \%$ of acute GI and GU toxicity based on registration with intraprostatic fiducial markers that allowed for smaller margins and subsequently lower acute toxicity to the bladder and rectum. All patients were given neoadjuvant hormonal therapy for $4-6$ weeks prior to radiation and concomitant with radiation therapy. There is evidence that androgen deprivation has favorable impacts on both local (prostate) disease and distant metastatic disease [36]. Mercader et al. [37] reported a T-cell infiltration of the prostate induced by ADT and a consequent increase in apoptosis. Furthermore, in an updated analysis of RTOG 94-13 phase III trial, Lawton et al. showed an unexpected interaction between $\mathrm{ADT}$ and radiation, which could be due to an immunomodulation in- 
duced by the $\mathrm{AD}$, resulting in improved PFS of patients with $15 \%$ risk of nodal involvement [4] [5].

The limitations of our study include the small number of patients in the analysis and the short follow-up period to assess the late toxicity of this technique. However, the acceptably low incidence of acute toxicity and absence of grade III toxicity would predict a low incidence of chronic toxicity. Furthermore, the role of combined dose escalation and ADT in high risk prostate cancer patients and its effect on overall survival (OS) needs to be assessed in the future by phase III randomized study.

\section{Conclusion}

The use of dose escalation in two phases combined with ADT in high risk prostate cancer patient is feasible and associated with acceptable acute GI and GU toxicity. Daily Image guidance is effective to ensure adequate coverage of both LNs and prostate with good sparing of risk organs.

\section{Ethics Approval and Consent to Participate}

Waiver of consent was granted by the local research ethics board of Fakeeh Hospital. As a retrospective study, individual patient consents were not obtained. Patient identifiers were not used.

\section{Conflicts of Interest}

The authors declare no conflicts of interest regarding the publication of this paper.

\section{References}

[1] Beckendorf, V., Guerif, S., Le Prise, E., et al. (2011) 70 Gy versus 80 Gy in Localized Prostate Cancer: 5-Year Results of GETUG 06 Randomized Trial. International Journal of Radiation Oncology, Biology, Physics, 80, 1056-1063. https://doi.org/10.1016/j.ijrobp.2010.03.049

[2] Zietman, A.L., DeSilvio, M.L., Slater, J.D., et al. (2005) Comparison of Conventional-Dose vs High-Dose Conformal Radiation Therapy in Clinically Localized Adenocarcinoma of the Prostate: A Randomized Controlled Trial. The Journal of the American Medical Association, 294, 1233-1239. https://doi.org/10.1001/jama.294.10.1233

[3] Al-Mamgani, A., van Putten, W.L., van der Wielen, G.J., Levendag, P.C. and Incrocci, L. (2011) Dose Escalation and Quality of Life in Patients with Localized Prostate Cancer Treated with Radiotherapy: Long-Term Results of the Dutch Randomized Dose-Escalation Trial (CKTO 96-10 Trial). International Journal of Radiation Oncology, Biology, Physics, 79, 1004-1012. https://doi.org/10.1016/j.ijrobp.2009.12.039

[4] Roach, M., De Silvio, M., Lawton, C., et al. (2003) Phase III Trial Comparing Whole-Pelvis versus Prostate-Only Radiotherapy and Neoadjuvant versus Adjuvant Combined Androgen Suppression: Radiation Therapy Oncology Group 9413. Journal of Clinical Oncology, 21, 1904-1911. https://doi.org/10.1200/JCO.2003.05.004 
[5] Lawton, C.A., De Silvio, M., Roach, M., et al. (2007) An Update of the Phase III Trial Comparing Whole Pelvic to Prostate Only Radiotherapy and Neoadjuvant to Adjuvant Total Androgen Suppression: Updated Analysis of RTOG 94-13, with Emphasis on Unexpected Hormone/Radiation Interactions. International Journal of Radiation Oncology, Biology, Physics, 69, 646-655. https://doi.org/10.1016/j.ijrobp.2007.04.003

[6] Pahlajani, N., Ruth, K.J., Buyyounouski, M.K., et al. (2012) Radiotherapy Doses of 80 Gy and Higher Are Associated with Lower Mortality in Men with Gleason Score 8 to 10 Prostate Cancer. International Journal of Radiation Oncology, Biology, Physics, 82, 1949-1956. https://doi.org/10.1016/j.ijrobp.2011.04.005

[7] Zelefsky, M.J., Pei, X., Chou, J.F., et al. (2011) Dose Escalation for Prostate Cancer Radiotherapy: Predictors of Long-Term Biochemical Tumor Control and Distant Metastases-Free Survival Outcomes. European Urology, 60, 1133-1139. https://doi.org/10.1016/j.eururo.2011.08.029

[8] Hennequin, C., Richaud, P.M., Roca, L., et al. (2015) Randomized Phase 3 Trial of Dose Escalation (80 vs 70 Gy) in High-Risk Prostate Cancers Combined with Long-Term Androgen Deprivation: GETUG-AFU 18 Trial, Acute and 1-Year Toxicities. International Journal of Radiation Oncology, Biology, Physics, 93, S44-S45. https://doi.org/10.1016/j.ijrobp.2015.07.107

[9] Sanguineti, G., Cavey, M.L., Endres, E.J., et al. (2006) Does Treatment of the Pelvic nodes with IMRT Increase Late Rectal Toxicity over Conformal Prostate-Only Radiotherapy to 76 Gy? Strahlentherapie und Onkologie, 182, 543-549. https://doi.org/10.1007/s00066-006-1586-9

[10] Wang-Chesebro, A., Xia, P., Coleman, J., Akazawa, C. and Roach, M, (2006) Intensity Modulated Radiotherapy Improves Lymph Node Coverage and Dose to Critical Structures Compared with Three-Dimensional Conformal Radiation Therapy in Clinically Localized Prostate Cancer. International Journal of Radiation Oncology, Biology, Physics, 66, 654-662. https://doi.org/10.1016/j.ijrobp.2006.05.037

[11] Sheets, N.C., Goldin, G.H., Meyer, A.M., et al. (2012) Intensity-Modulated Radiation Therapy, Proton Therapy, or Conformal Radiation Therapy and Morbidity and Disease Control in Localized Prostate Cancer. The Journal of the American Medical Association, 307, 1611-1620. https://doi.org/10.1001/jama.2012.460

[12] Muren, L.P., Wasbø, E., Helle, S.I., et al. (2008) Intensity-Modulated Radiotherapy of Pelvic Lymph Nodes in Locally Advanced Prostate Cancer: Planning Procedures and Early Experiences. International Journal of Radiation Oncology, Biology, Physics, 71, 1034-1041. https://doi.org/10.1016/j.ijrobp.2007.11.060

[13] Pervez, N., Small, C., MacKenzie, M., et al. (2010) Acute Toxicity in Highrisk Prostate Cancer Patients Treated with Androgen Suppression and Hypofractionated Intensity-Modulated Radiotherapy. International Journal of Radiation Oncology, Biology, Physics, 76, 57-64. https://doi.org/10.1016/j.ijrobp.2009.01.048

[14] Bayley, A., Rosewall, T., Craig, T., et al. (2010) Clinical Application of High-Dose, Image-Guided Intensity-Modulated Radiotherapy in High-Risk Prostate Cancer. International Journal of Radiation Oncology, Biology, Physics, 77, 477-483. https://doi.org/10.1016/j.ijrobp.2009.05.006

[15] Guckenberger, M., Baier, K., Richter, A., Vordermark, D. and Flentje, M. (2008) Does Intensity Modulated Radiation Therapy (IMRT) Prevent Additional Toxicity of Treating the Pelvic Lymph Nodes Compared to Treatment of the Prostate Only? Radiation Oncology, 3, 3. https://doi.org/10.1186/1748-717X-3-3

[16] Zhang, P., Happersett, L., Hunt, M., et al. (2010) Volumetric Modulated Arc Ther- 
apy: Planning and Evaluation for Prostate Cancer Cases. International Journal of Radiation Oncology, Biology, Physics, 76, 1456-1462. https://doi.org/10.1016/j.ijrobp.2009.03.033

[17] Aznar, M.C., Petersen, P.M., Logadottir, A., et al. (2010) Rotational Radiotherapy for Prostate Cancer in Clinical Practice. Radiotherapy and Oncology, 97, 480-484. https://doi.org/10.1016/j.radonc.2010.09.014

[18] Kopp, R.W., Duff, M., Catalfamo, F., et al. (2011) VMAT vs. 7-Field-IMRT: Assessing the Dosimetric Parameters of Prostate Cancer Treatment with a 292-Patient Sample. Medical Dosimetry, 36, 365-372. https://doi.org/10.1016/j.meddos.2010.09.004

[19] Edge, S.B. and Compton, C.C. (2010) The American Joint Committee on Cancer: The 7th Edition of the AJCC Cancer Staging Manual and the Future of TNM. Annals of Surgical Oncology, 17, 1471-1474. https://doi.org/10.1245/s10434-010-0985-4

[20] Lawton, C.A.F., Michalski, J., El-Naqa, I., et al. (2009) RTOG GU Radiation Oncology Specialists Reach Consensus on Pelvic Lymph Node Volumes for High-Risk Prostate Cancer. International Journal of Radiation Oncology, Biology, Physics, 74, 383-387. https://doi.org/10.1016/j.ijrobp.2008.08.002

[21] Gay, H.A., Barthold, H.J., O’Meara, E., et al. (2012) Pelvic Normal Tissue Contouring Guidelines for Radiation Therapy: A Radiation Therapy Oncology Group Consensus Panel Atlas. International Journal of Radiation Oncology, Biology, Physics, 83, e353-e362. https://doi.org/10.1016/j.ijrobp.2012.01.023

[22] Morikawa, L.K. and Roach, M. (2011) Pelvic Nodal Radiotherapy in Patients with Unfavorable Intermediate and High-Risk Prostate Cancer: Evidence, Rationale, and Future Directions. International Journal of Radiation Oncology, Biology, Physics, 80, 6-16. https://doi.org/10.1016/j.ijrobp.2010.11.074

[23] Pommier, P., Chabaud, S., Lagrange, J.L., et al. (2007) Is There a Role for Pelvic Irradiation in Localized Prostate Adenocarcinoma? Preliminary Results of GETUG-01. Journal of Clinical Oncology, 25, 5366-5373. https://doi.org/10.1200/JCO.2006.10.5171

[24] Radiation Therapy Oncology Group (2011) Androgen Deprivation Therapy and High Dose Radiotherapy with or without Whole-Pelvic Radiotherapy in Unfavorable Intermediate or Favorable High Risk Prostate Cancer: A Phase III Randomized Trial.

[25] Kaidar-Person, O., Roach, M. and Crehange, G. (2013) Whole-Pelvic Nodal Radiation Therapy in the Context of Hypofractionation for High-Risk Prostate Cancer Patients: A Step forward. International Journal of Radiation Oncology, Biology, Physics, 86, 600-605. https://doi.org/10.1016/j.ijrobp.2013.02.006

[26] Yoo, S., Wu, Q.J., Lee, W.R. and Yin, F.-F. (2010) Radiotherapy Treatment Plans with Rapid Arc for Prostate Cancer Involving Seminal Vesicles and Lymph Nodes. International Journal of Radiation Oncology, Biology, Physics, 76, 935-942. https://doi.org/10.1016/j.ijrobp.2009.07.1677

[27] Riou, O., Mothe, P.R., Azria, D., et al. (2013) Simultaneous Integrated Boost Plan Comparison of Volumetric-Modulated Arc Therapy and Sliding Window Intensity-Modulated Radiotherapy for Whole Pelvis Irradiation of Locally Advanced Prostate Cancer. Journal of Applied Clinical Medical Physics, 14, 26-35. https://doi.org/10.1120/jacmp.v14i4.4094

[28] Deville, C., Both, S., Hwang, W.T., Tochner, Z. and Vapiwala, N. (2010) Clinical Toxicities and Dosimetric Parameters after Whole-Pelvis versus Prostate-Only In- 
tensity-Modulated Radiation Therapy for Prostate Cancer. International Journal of Radiation Oncology, Biology, Physics, 78, 763-772. https://doi.org/10.1016/j.ijrobp.2009.08.043

[29] Ishii, K., Ogino, R., Hosokawa, Y., et al. (2015) Whole-Pelvic Volumetric-Modulated Arc Therapy for High-Risk Prostate Cancer: Treatment Planning and Acute Toxicity. Journal of Radiation Research, 56, 141-150.

https://doi.org/10.1093/jrr/rru086

[30] Vargas, C., Martinez, A., Kestin, L.L., et al. (2005) Dose-Volume Analysis of Predictors for Chronic Rectal Toxicity after Treatment of Prostate Cancer with Adaptive Image-Guided Radiotherapy. International Journal of Radiation Oncology, Biology, Physics, 62, 1297-308. https://doi.org/10.1016/j.ijrobp.2004.12.052

[31] Michalski, J.M., Yan, Y., Watkins-Bruner, D., et al. (2013) Preliminary Toxicity Analysis of 3-Dimensional Conformal Radiation Therapy versus Intensity Modulated Radiation Therapy on the High-Dose Arm of the Radiation Therapy Oncology Group 0126 Prostate Cancer Trial. International Journal of Radiation Oncology, Biology, Physics, 87, 932-938. https://doi.org/10.1016/j.ijrobp.2013.07.041

[32] De Meerleer, G., Vakaet, L., Meersschout, S., et al. (2004) Intensitymodulated Radiotherapy as Primary Treatment for Prostate Cancer: Acute Toxicity in 114 Patients. International Journal of Radiation Oncology, Biology, Physics, 60, 777-787. https://doi.org/10.1016/j.ijrobp.2004.04.017

[33] Arcangeli, S., Saracino, B., Grazia, M., et al. (2007) Analysis of Toxicity in Patients with High Risk Prostate Cancer Treated with Intensitymodulated Pelvic Radiation Therapy and Simultaneous Integrated Dose Escalation to Prostate Area. Radiotherapy and Oncology, 84, 148-155. https://doi.org/10.1016/j.radonc.2007.06.011

[34] Ferjani, S., Huang, G., Shang, Q., et al. (2013) Alignment Focus of Daily Image Guidance for Concurrent Treatment of Prostate and Pelvic Lymph Nodes. International Journal of Radiation Oncology, Biology, Physics, 87, 383-389. https://doi.org/10.1016/j.ijrobp.2013.06.003

[35] Chung, H.T., Xia, P., Chan, L.W., Park-Somers, E. and Roach, M. (2009) Does Image-Guided Radiotherapy Improve Toxicity Profile in Whole Pelvic-Treated High-Risk Prostate Cancer? Comparison between IG-IMRT and IMRT. International Journal of Radiation Oncology, Biology, Physics, 73, 53-60. https://doi.org/10.1016/j.ijrobp.2008.03.015

[36] Soloway, M.S., Sharifi, R., Wajsman, Z., McLeod, D., Wood Jr., D.P. and Puras-Baez, A. (1995) Randomized Prospective Study Comparing Radical Prostatectomy alone versus Radical Prostatectomy Preceded by Androgen Blockade in Clinical Stage B2 (T2bNxM0) Prostate Cancer. The Lupron Depot Neoadjuvant Prostate Cancer Study Group. Journal of Urology, 154, 424-428. https://doi.org/10.1016/S0022-5347(01)67067-8

[37] Mercader, M., Bodner, B.K., Moser, M.T., Kwon, P.S., Park, E.S., Manecke, R.G., et al. 2001. T-Cell Infiltration of the Prostate Induced by Androgen Withdrawal in $\mathrm{Pa}$ tients with Prostate Cancer. Proceedings of the National Academy of Sciences of the United States of America, 98, 14565-14570. https://doi.org/10.1073/pnas.251140998 


\section{Abbreviations}

ADT: Androgen Deprivation Therapy;

CTV: Clinical Target Volume;

CTCAE: Common Terminology Criteria for Adverse Events;

DVHs: Dose Volume Histograms;

IMRT: Intensity Modulated Radiation Therapy;

GI: Gastrointestinal;

GU: Genitourinary;

OAR: Organs at Risk;

OS: Overall Survival;

PFS: Progression Free Survival;

PORT: Prostate Only Radiation Therapy;

PSA: Prostatic Specific Antigen;

VMAT: Volumetric Modulated Arc Therapy;

WP: Whole Pelvis;

(3-DCRT): 3-Dimintional Conformal Radiation Therapy. 